\title{
Improving Pancreatic Islet Engraftment after Islet Transplantation through Administration of Gamma-Secretase Inhibitor DAPT
}

\author{
Daisy Hjelmqvist ${ }^{1}$, Mats Hellström² ${ }^{2}$ and Joey Lau ${ }^{1, *}$ \\ ${ }^{1}$ Department of Medical Cell Biology, BMC, Uppsala University, 75123 Uppsala, Sweden \\ ${ }^{2}$ Department of Immunology, Genetics and Pathology, Science for Life Laboratory, Rudbeck Laboratory, \\ Uppsala University, 75185 Uppsala, Sweden
}

\begin{abstract}
Rapid and effective revascularization of transplanted pancreatic islets is vital for the survival and function of the islet graft. Insufficient vascularization after islet transplantation may be one causative factor to the failure of islet grafts in clinical transplantation. The aim of this study was to investigate if $N$ - $N$-[2-(3,5-Difluorophenyl)acetyl]-(S)-alanyl\}(S)-phenylglycine- tert-butyl ester (DAPT) administration can improve engraftment of transplanted islets. DAPT is a dipeptidic gamma-secretase inhibitor which inhibits Notch signaling. Notch signaling is involved in angiogenesis and inhibition may result in excessive formation of new blood vessels. Excessive vasculature may be beneficial in the immediate posttransplantation period since the transplanted islets are dependent on diffusion of oxygen and nutrients before revascularization.

Islets isolated from C57BL/6 mice were transplanted beneath the renal capsule of C57BL/6 mice. After islet transplantation DAPT or vehicle was administered subcutaneously for three days.

Mice treated with DAPT had an increased vascular density when compared to control mice two days and one month posttransplantation. Moreover, mice treated with DAPT showed $54 \pm 8.2 \%$ functional blood vessels compared to $40 \pm 6.7$ $\%$ in control mice two days posttransplantation. After one month, the fraction of functional blood vessels increased to $86 \pm 2.8 \%$ in DAPT treated mice compared to $61 \pm 9.4 \%$ in control mice.
\end{abstract}

Our findings demonstrated that administration of DAPT may be a feasible strategy to improve engraftment of transplanted islets.

Keywords: Islets of Langerhans, islet transplantation, vascular density, gamma-secretase inhibitor, DAPT.

\section{INTRODUCTION}

Type 1 diabetes mellitus (T1D) is a disease characterized by elevated blood glucose levels (hyperglycemia) which results from the decreased or absent action of insulin secretion. T1D is also called insulin-dependent diabetes mellitus which generally results from autoimmune destruction of beta-cells in the pancreatic islet with consequent insulin deficiency and requirement of exogenous insulin treatment [1].

Pancreatic islet transplantation into the liver has evolved into a promising treatment option for a subgroup of type 1 diabetes patients [2]. It is an emerging alternative to whole pancreas transplantation. However, several hurdles restrict the widespread application of this approach such as shortage of donor organs; more than one donor per recipient is needed to reach insulin-independence, preservation of islets during isolation, gradual loss in islet graft function due to inadequate oxygen supply and insufficient revascularization. Moreover, there is a constant need for immunosuppression which is linked with potentially

*Address correspondence to this author at the Department of Medical Cell Biology, Uppsala University, Husargatan 3, Box 571, SE-75123 Uppsala, Sweden; Tel: +46 18 4714395; Fax: +46 18 4714059;

E-mail: Joey.Lau@mcb.uu.se severe adverse effect and which may impair regeneration and revascularization of the transplanted islets. Albeit, this technique is minimally invasive in comparison to whole pancreas transplantation that requires open surgery and general anesthesia [3]. Several therapeutics strategies have been developed to improve engraftment of the transplanted islets such as islet microencapsulation using different biosynthetic materials. But microencapsulation of islets has had limited clinical success due to fibroblast overgrowth, insufficient oxygen supply which subsequently causes loss of islet mass, function and cell death [4]. Therefore, there is a need of alternative methods to enhance the survival, function and engraftment of transplanted islets.

One possible way to increase vascularization of grafted islets is to block endogenous inhibitors of angiogenesis. The Notch signaling is involved in angiogenesis and other cellular processes. Total block of Notch signaling during vascular development results in excessive formation of new blood vessels [5]. Recent studies have shown that specific inhibition of DII4/Notch reduced tumor growth through increasing the vascular density in the tumor. Excessive branching and sprouting resulted in an extremely disordered vascular network that lacked the system necessary for efficient 
directional blood flow $[6,7]$. It has also been shown that indirect inhibition of Notch signaling via blockade of the protease complex gamma-secretase using low molecular weight inhibitor can affect the promotion of excessive non-productive angiogenesis and lead to tumor growth inhibition [8]. Another study showed that low dosage inhibition of Dll4/Notch signaling led to improved vascular function and accelerated wound healing [7]. Moreover, Cao et al. demonstrated that modulation of Notch signaling through local and sustained administration of the gamma-secretase inhibitor DAPT and dose dependent VEGF enhanced neovascularization and perfusion recovery in diabetic mice suffering from ischemia [9].

In this study, $N-\{N$-[2-(3,5-Difluorophenyl)acetyl](S)-alanyl\}-(S)-phenylglycine-tert-butyl ester (DAPT), a dipeptidic gamma-secretase inhibitor was investigated. DAPT indirectly inhibits Notch which is a gammasecretase substrate [10]. The aim of this study was to investigate if systemic delivery of DAPT can improve engraftment of transplanted islets through the stimulation of angiogenesis.

\section{RESEARCH DESIGN AND METHODS}

\section{Animals}

All experiments were approved by the Animal Ethical Committee in Uppsala. Adult, male C57BL/6 mice were purchased from Taconic (Ry, Denmark).

\section{Islet Isolation and Culture}

Pancreatic islets of C57BL/6 mice were isolated using collagenase digestion and density gradient purification [11]. Islets were thereafter handpicked and cultured free-floating in RPMI 1640 (Sigma-Aldrich, St. Louis, MO) supplemented with L-glutamine $(2 \mathrm{mmol} / \mathrm{l}$; Sigma-Aldrich), benzylpenicillin (100 U/ml; Roche Diagnostics, Bromma, Sweden), streptomycin (0.1 $\mathrm{mg} / \mathrm{ml}$; Sigma-Aldrich) and $10 \%$ (vol/vol) fetal calf serum (Sigma-Aldrich) at $37^{\circ} \mathrm{C}\left(\mathrm{O}_{2} / \mathrm{CO}_{2}, 95: 5\right)$.

\section{Islet Transplantation and Administration of DAPT/Vehicle}

100 islets were packed in a braking pipette and implanted beneath the renal capsule of C57BL/6 mice that had been anesthetized with avertin [a 2.5\% ( $\mathrm{vol} / \mathrm{vol})$ solution of $10 \mathrm{~g} 97 \%$ ( $\mathrm{vol} / \mathrm{vol}$ ) 2,2,2-tribromoethanol (Sigma-Aldrich) in $10 \mathrm{ml}$ of 2-methyl-2-butanol (Kemila, Stockholm, Sweden)]. Low dose DAPT (1 mg/kg; \#565770, Merk Millipore, Darmstadt, Germany) or vehicle $(90 \%$ corn oil $+10 \%$ ethanol) was injected subcutaneously after transplantation (day 0 ) and at day 1 and 2.

\section{Perfusion of Graft Bearing Mice}

Two days and one month posttransplantation, 100 $\mu \mathrm{l}$ of $1 \mathrm{mg} / \mathrm{ml} \mathrm{FITC}$ conjugated (tomato) lectin (Vector Laboratories, Burlingame, CA, USA) was given intravenously through the tail vein of each islet graft bearing C57BL/6 mouse. 20 minutes later mice were anesthetized with avertin before perfusion fixation with $4 \%$ paraformaldehyde solution. The islet graft bearing kidneys were then removed and post-fixated with $4 \%$ paraformaldehyde at $4^{\circ} \mathrm{C}$ overnight. Following fixation, the graft bearing kidneys were washed with cold PBS for one hour and then equilibrated in $15 \%$ sucrose in PBS for two hours and then $30 \%$ sucrose in PBS overnight at $4^{\circ} \mathrm{C}$. Thereafter, the graft bearing kidneys were embedded in frozen section medium (RichardAllan Scientific NEG 50, Thermo Scientific, Kalamazoo, MI, USA) in cryomolds (Tissue-Tek Cryomold, Sakura Finetek Inc, Torrance, CA, USA), frozen on dry ice and stored in the $-80{ }^{\circ} \mathrm{C}$ freezer.

\section{Immunohistochemistry}

Cryosections of each islet graft bearing kidney ( 7 $\mu \mathrm{m}$ thick) were mounted on polysine coated glass slides (Thermo Scientific) and stored in the $-80{ }^{\circ} \mathrm{C}$ freezer. Sections were dried on heater at $40^{\circ} \mathrm{C}$ for 10 min, equilibrated in PBS for $3 \mathrm{~min}$ and blocked with PBS containing 1\% Bovine Serum Albumin (SigmaAldrich), $0.3 \%$ Triton $\mathrm{X} 100$ and $0.1 \% \mathrm{NaN}_{3}$ for one hour at room temp (RT). Sections were incubated with primary antibodies (polyclonal guinea pig anti-insulin, 1:300 (Dako, Glostrup, Denmark) and monoclonal rat anti-mouse CD31, 1:50 (clone MEC 13.3, BD Pharmingen, San Diego, CA, USA)) for one hour in RT following incubation with secondary antibodies (Alexa Fluor 555 goat anti rat $\operatorname{lgG}$ and Alexa Fluor 633 goat anti guinea pig IgG, 1:200, Invitrogen, Eugene, Oregon, USA) incubated for $20 \mathrm{~min}$ in RT. The glass slides were mounted with Prolong Gold with DAPI (Invitrogen).

\section{Analysis of Vascular Density}

The stained islet graft sections were scanned with a Laser Scanning Microscope ZEISS LSM780. All shown images have been subjected to a median filter and changes in brightness and contrast for optimal visualization.

The scanned images were analyzed with IMARIS ${ }^{\circledR}$ 7.6.1 (BITPLANE Scientific Software). The vascular 
density was defined as CD31 positive structures per islet insulin positive area. The degree of perfused blood vessels was defined as FITC-lectin positive structures of total CD31 positive structures.

\section{Statistical Analysis}

Values are expressed as means \pm SEM for 4-6 animals in each group. All statistical analysis was made with Mann Whitney rank sum test. For all comparisons, $\mathrm{P}<0.05$ was considered statistically significant.

\section{RESULTS AND DISCUSSION}

Survival and function of islet graft depends on the rapid revascularization and sufficient oxygen supply. Effective revascularization requires a delicate balance between branching and sprouting of new blood vessels and maintenance of the developing vascular tube. In the present study, we demonstrated that subcutaneous injection of the Notch inhibitor, DAPT, increased the vascular density and the number of perfused vessels of transplanted islets in mice.

We assessed different doses of DAPT in mice transplanted with islets beneath the renal capsule; 1 $\mathrm{mg} / \mathrm{kg}(\mathrm{n}=3), 10 \mathrm{mg} / \mathrm{kg}(\mathrm{n}=3)$ and $100 \mathrm{mg} / \mathrm{kg}(\mathrm{n}=2)$, (data not shown). We found most blood vessels in the low dose DAPT (1 mg/kg) treated group. We, therefore, decided to continue with the low dose DAPT treatment. The vascular density in islet grafts treated with DAPT was increased when compared to control islet grafts both two days (Figure 1A) and one month posttransplantation (Figure 1B).

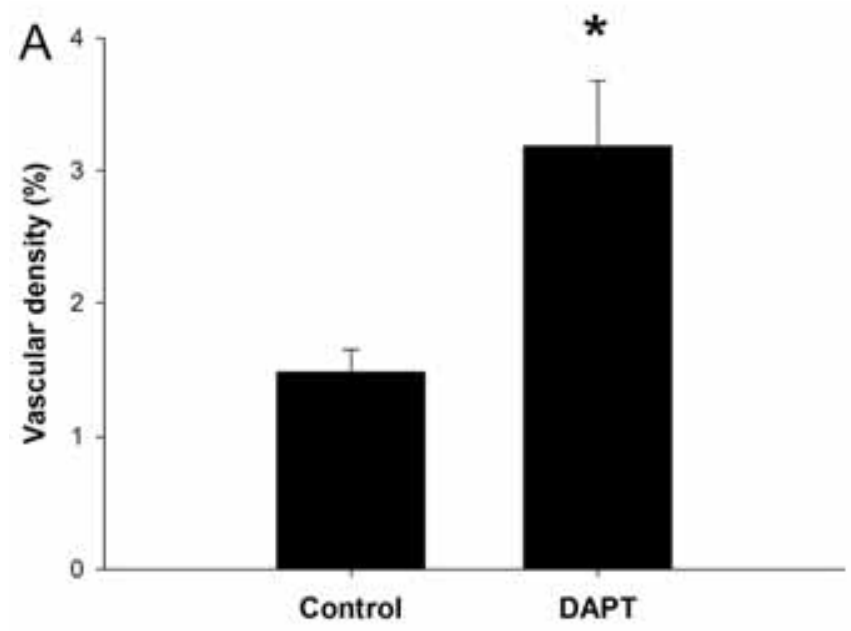

We chose the early time point, two days posttransplantation, to investigate the effect of DAPT when the revascularization process begins (Figure 2AD). Revascularization of transplanted islets is completed two weeks posttransplantation, we chose one month as final end point (Figure 2E-H).

Although few blood vessels were found within the transplanted islets two days posttransplantation, numerous blood vessels were discerned in the periphery of transplanted islets in the DAPT treated group, which probably contributes to improved oxygen supply to the transplanted islets. Due to acute factors like hypoxia and inflammatory reactions, up to $60 \%$ of the transplanted islets will undergo necrosis and apoptosis early after transplantation [12-15]. The delivery of oxygen is therefore critical in the immediate phase after islet transplantation, thus loss of islet mass can be prevented. We assessed the functionality of the blood vessels by FITC-conjugated lectin injection. The great increase in vascular density one month posttransplantation in mice treated with DAPT showed also an increase in functional blood vessels compared to control mice $(86 \pm 2.8 \%$ vs. $61 \pm 9.4 \%$, respectively, $\mathrm{p}<0.05)$. Two days posttransplantation, the fraction of functional blood vessels was $54 \pm 8.2 \%$ in DAPT treated mice compared to $40 \pm 6.7 \%$ in control mice $(p=0.247)$.

Subcutaneous injection of DAPT after islet transplantation showed an increased vascular density when compared with control islets. These results demonstrate that administration of DAPT has the potential as a promising approach for preventing loss of transplanted islets due to hypoxia-induced cell death by providing a rapid revascularization of the islet graft.

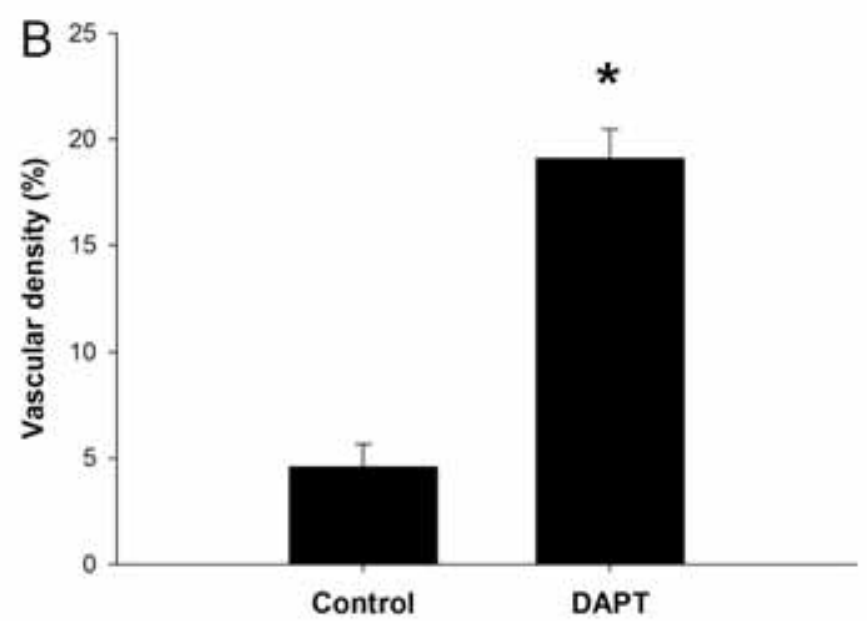

Figure 1: Vascular density in islets transplanted beneath the renal capsule of C57BL/6 mice. Islet grafts consisting of control and low dose DAPT $(1 \mathrm{mg} / \mathrm{kg})$ two days posttransplantation $(\mathbf{A})$ and one month posttransplantation (B). All values are expressed as means \pm SEM for 4-6 animals in each group. * denotes $P<0.05$. 

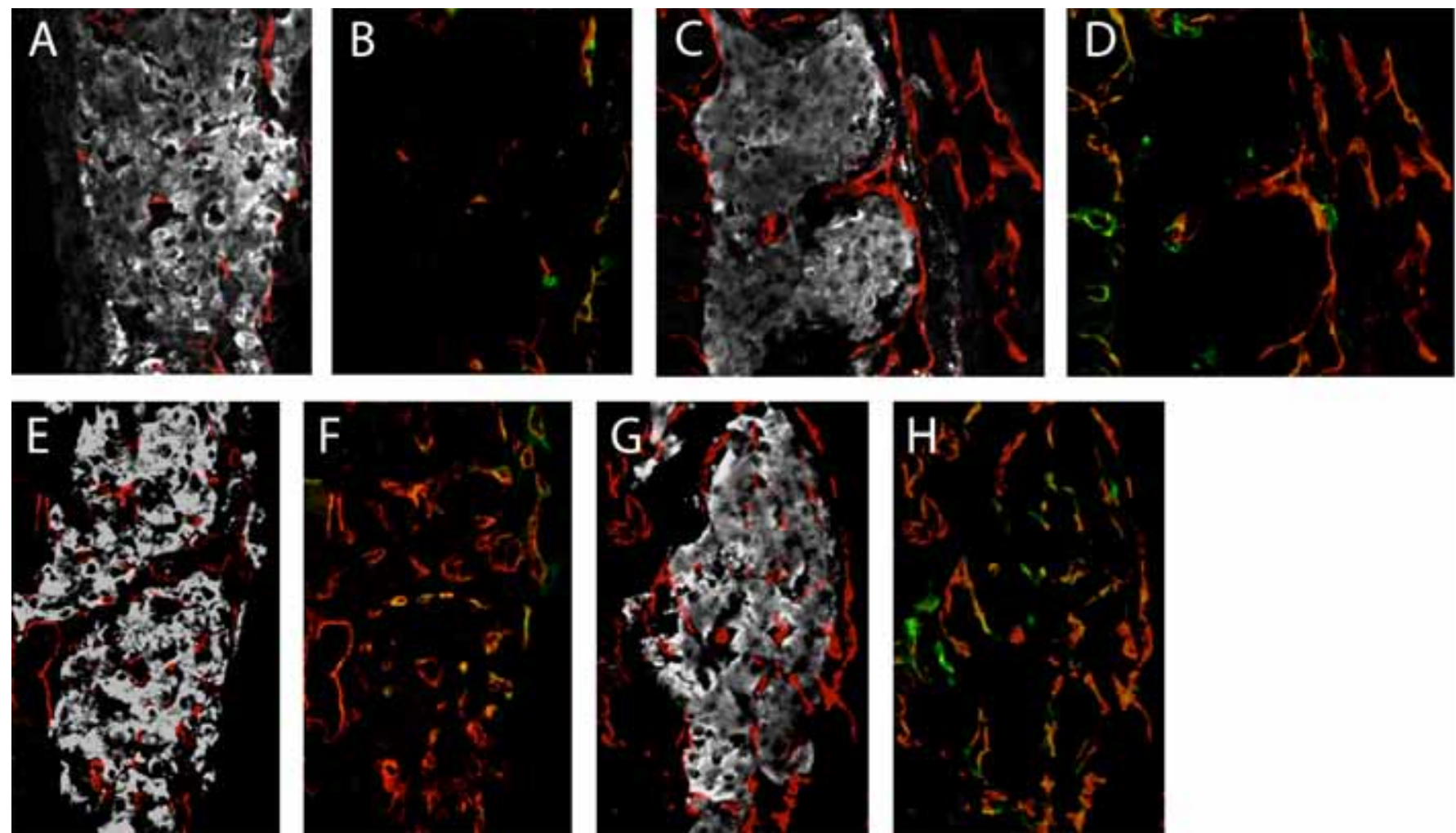

Figure 2: Confocal images of control islet graft (A-B) (vehicle $90 \%$ corn oil $+10 \%$ ethanol) and low dose DAPT (1 mg/kg) treated islet graft (C-D) two days posttransplantation. Confocal images (E-F) and (G-H) show control islet graft and low dose DAPT treated islet graft one month posttransplantation, respectively. Grey represents insulin $(\mathbf{A}, \mathbf{C}, \mathbf{E}$ and $\mathbf{G})$; red represents CD31 staining for blood vessels $(\mathbf{A}-\mathbf{H})$ and green represents FITC-conjugated lectin $(\mathbf{B}, \mathbf{D}, \mathbf{F}$ and $\mathbf{H})$. Overlay images of insulin and CD31 are shown in $\mathbf{A}, \mathbf{C}, \mathbf{E}$ and $\mathbf{G}$ whereas the overlay images $\mathbf{B}, \mathbf{D}, \mathbf{F}$ and $\mathbf{H}$ show CD31 and FITC-conjugated lectin.

\section{CONCLUSIONS}

Administration of DAPT for three days demonstrated an increased vascular density when compared with control at both time points i.e. two days and one month posttransplantation. Our findings demonstrate that administration of gamma-secretase inhibitors may be a feasible strategy to improve engraftment of the transplanted islets. Future studies should focus on the specific blockade of Notch signaling components (i.e. DII4 or Notch1/4) and the effect of gamma-secretase/Notch inhibition on the survival and function of the engrafted islets.

\section{ACKNOWLEDGEMENTS}

The study was generously supported by the Swedish Juvenile Diabetes Foundation, the Swedish Society of Medicine, the Fredrik and Ingrid Thuring Foundation, Magnus Bergvall Foundation, Swedish Cancer Foundation and Lars Hiertas Memorial Foundation. We gratefully thank Anja Nitzsche for preparing DAPT and vehicle used in the study and Svitlana Vasylovska for skilled assistance in perfusion fixation of transplanted mice.

\section{REFERENCES}

[1] Atkinson MA, Eisenbarth GS. Type 1 diabetes: new perspectives on disease pathogenesis and treatment. Lancet 2001; 358: 221-9.

http://dx.doi.org/10.1016/S0140-6736(01)05415-0

[2] Shapiro AM, Lakey JR, Ryan EA, et al. Islet transplantation in seven patients with type 1 diabetes mellitus using a glucocorticoid-free immunosuppressive regimen. $\mathrm{N}$ Engl $\mathrm{J}$ Med 2000; 343: 230-8

http://dx.doi.org/10.1056/NEJM200007273430401

[3] Langer RM. Islet transplantation: lessons learned since the Edmonton breakthrough. Transplant Proc 2010; 42: 1421-4 http://dx.doi.org/10.1016/j.transproceed.2010.04.021

[4] Ludwig B, Rotem A, Schmid J, et al. Improvement of islet function in a bioartificial pancreas by enhanced oxygen supply and growth hormone releasing hormone agonist. Proc Natil Acad Sci USA 2012; 109: 5022-7. http://dx.doi.org/10.1073/pnas.1201868109

[5] Hellstrom M, Phng LK, Hofmann JJ, et al. DIl4 signalling through Notch1 regulates formation of tip cells during angiogenesis. Nature 2007; 445: 776-80.

http://dx.doi.org/10.1038/nature05571

[6] Yan M, Plowman GD. Delta-like 4/Notch signaling and its therapeutic implications. Clin Cancer Res 2007; 13: 7243-6. http://dx.doi.org/10.1158/1078-0432.CCR-07-1393

[7] Trindade A, Djokovic D, Gigante J, et al. Low-dosage inhibition of DIl4 signaling promotes wound healing by inducing functional neo-angiogenesis. PloS One 2012; 7: e29863.

http://dx.doi.org/10.1371/journal.pone.0018709 
[8] Kalen M, Heikura $\mathrm{T}$, Karvinen $\mathrm{H}$, et al. Gamma-secretase inhibitor treatment promotes VEGF-A-driven blood vessel growth and vascular leakage but disrupts neovascular perfusion. PloS One 2011; 6: e18709.

[9] Cao L, Arany PR, Kim J, et al. Modulating Notch signaling to enhance neovascularization and reperfusion in diabetic mice. Biomaterials 2010; 31: 9048-56.

http://dx.doi.org/10.1016/j.biomaterials.2010.08.002

[10] Czerwinski A, Valenzuela F, Afonine P, Dauter M, Dauter Z. $\mathrm{N}-\{\mathrm{N}-[2-(3,5-$ Difluorophenyl)acetyl]-(S)-alanyl\}-(S)phenylglycine tert-butyl ester (DAPT): an inhibitor of gammasecretase, revealing fine electronic and hydrogen-bonding features. Acta Crystallogr C. 2010; 66(Pt 12): 585-8. http://dx.doi.org/10.1107/S0108270110044136

[11] Henriksnas J, Lau J, Zang G, Berggren PO, Kohler M, Carlsson PO. Markedly decreased blood perfusion of pancreatic islets transplanted intraportally into the liver: disruption of islet integrity necessary for islet revascularization. Diabetes 2012; 61: 665-73. http://dx.doi.org/10.2337/db10-0895
[12] Biarnes $M$, Montolio $M$, Nacher $V$, Raurell $M$, Soler $J$, Montanya E. Beta-cell death and mass in syngeneically transplanted islets exposed to short- and long-term hyperglycemia. Diabetes 2002; 51: 66-72.

http://dx.doi.org/10.2337/diabetes.51.1.66

[13] Emamaullee JA, Shapiro AM. Factors influencing the loss of beta-cell mass in islet transplantation. Cell Transplant 2007; 16: 1-8.

[14] Carlsson PO, Palm F, Andersson A, Liss P. Markedly decreased oxygen tension in transplanted rat pancreatic islets irrespective of the implantation site. Diabetes 2001; 50: 489-95. http://dx.doi.org/10.2337/diabetes.50.3.489

[15] Eich $T$, Eriksson O, Sundin A, et al. Positron emission tomography: a real-time tool to quantify early islet engraftment in a preclinical large animal model. Transplantation 2007; 84: 893-8. http://dx.doi.org/10.1097/01.tp.0000284730.86567.9f

(C) 2014 Hjelmqvist et al.; Licensee Synergy Publishers.

This is an open access article licensed under the terms of the Creative Commons Attribution Non-Commercial License (http://creativecommons.org/licenses/by-nc/3.0/) which permits unrestricted, non-commercial use, distribution and reproduction in any medium, provided the work is properly cited. 